\title{
Pulmonary tumor thrombotic microangiopathy caused by lung adenocarcinoma: Case report with review of the literature
}

\author{
MACHIKO HOTTA $^{1 *}$, MITSUAKI ISHIDA $^{1 *}$, FUMIYOSHI KOJIMA $^{1}$, YASUHIRO IWAI $^{2}$ and HIDETOSHI OKABE $^{1}$ \\ ${ }^{1}$ Department of Clinical Laboratory Medicine and Division of Diagnostic Pathology, \\ Shiga University of Medical Science, Shiga; ${ }^{2}$ Division of Diagnostic Pathology, Takatsuki Hospital, Osaka, Japan
}

Received December 20, 2010; Accepted March 4, 2011

DOI: $10.3892 / \mathrm{ol} .2011 .270$

\begin{abstract}
Pulmonary tumor thrombotic microangiopathy (PTTM) is an uncommon cancer-related complication characterized by intimal proliferation in pulmonary small arteries and arterioles with or without tumor emboli. In the majority of cases, the causative lesion is gastric poorly differentiated adenocarcinoma. In the present study, an autopsy case of PTTM caused by lung adenocarcinoma is reported and the pathogenesis of this complication is discussed. Multiple nodular lesions in the bilateral lungs were found in a 62-yearold Japanese man. Transbronchial biopsy revealed non-small cell carcinoma. Chemotherapy was performed; however, the patient succumbed to sudden dyspnea. Autopsy revealed poorly differentiated adenocarcinoma with multiple intrapulmonary metastases and intimal proliferation of pulmonary small arteries and arterioles with or without tumor emboli, which were characteristic of PTTM. Tumor cells were immunohistochemically positive for vascular endothelial growth factor (VEGF) and osteopontin (OPN), which are endothelial proliferative factors. This case indicates the possible involvement of VEGF and OPN in the pathogenesis of PTTM caused by lung adenocarcinoma.
\end{abstract}

\section{Introduction}

Pulmonary tumor thrombotic microangiopathy (PTTM), which was established as a distinct clinicopathological entity by von Herbay et al in 1990, is an uncommon cancer-related complication (1). Its primary histopathological characteristic is intimal proliferation in pulmonary small arteries and arterioles with or without tumor emboli, resulting in vascular stenosis.

Correspondence to: Dr Mitsuaki Ishida, Department of Clinical Laboratory Medicine and Division of Diagnostic Pathology, Shiga University of Medical Science, Tsukinowa-cho, Seta, Otsu, Shiga 520-2192, Japan

E-mail: mitsuaki@belle.shiga-med.ac.jp

*Contributed equally

Key words: lung cancer, osteopontin, pulmonary tumor thrombotic microangiopathy, vascular endothelial growth factor
In the majority of cases, the causative lesion is gastric poorly differentiated adenocarcinoma, including signet ring cell carcinoma (1-3). PTTM caused by lung cancer is extremely rare $(1,4)$

In the present report, an autopsy case of PTTM caused by lung adenocarcinoma is examined and the pathogenesis of this complication is discussed.

\section{Patients and methods}

A 62-year-old Japanese man with a history of cigarette smoking (30 years; one pack/day) presented with a persistent cough and shortness of breath lasting 1 year. A chest computed tomography (CT) showed multiple small nodules in the bilateral lungs (Fig. 1). Laboratory tests revealed elevated serum CA19-9 $(5,455 \mathrm{U} / \mathrm{ml}$; range <37) and carcinoembryonic antigen $(4,306 \mathrm{ng} / \mathrm{ml}$; range $<5)$. Transbronchial biopsy from the right lung nodule revealed non-small cell carcinoma and an abdominal CT showed metastases in the bilateral adrenal glands and lumbar vertebrae. The patient subsequently received four cycles of chemotherapy (cisplatin and vinorelbine), following which the above-mentioned tumor markers decreased (CA19-9, $692 \mathrm{U} / \mathrm{ml}$ and CEA, $823 \mathrm{ng} / \mathrm{ml}$ ). However, 5 months after the initial diagnosis two nodular lesions were found in S7 and S8 of the liver by abdominal CT. The lesions were diagnosed as metastases from lung carcinoma by clinical imaging findings. As a result, four cycles of chemotherapy (docetaxel) were added. Nine months after the initial diagnosis, the patient complained of a cough and shortness of breath again, and a chest CT showed an increase in the size and number of lung nodules. Radiation therapy was scheduled; however, the patient succumbed to sudden dyspnea.

Formalin-fixed, paraffin-embedded tissue blocks were cut into 3- $\mu \mathrm{m}$ sections, deparaffinized and rehydrated. Each section was stained with H\&E and Alcian blue, and used for immunohistochemical analyses. Immunohistochemical analyses were performed using an autostainer (XT system Benchmark; Ventana Medical System, Tucson, AZ, USA) according to the manufacturer's instructions. Primary antibodies were used against the following antigens: vascular endothelial growth factor (VEGF; A-20; Santa Cruz Biotechnology, Santa Cruz, CA, USA) and osteopontin (OPN; 10A16; Immuno-Biological Laboratories, Japan). The present study was approved by the Ethics Committee of our university. 


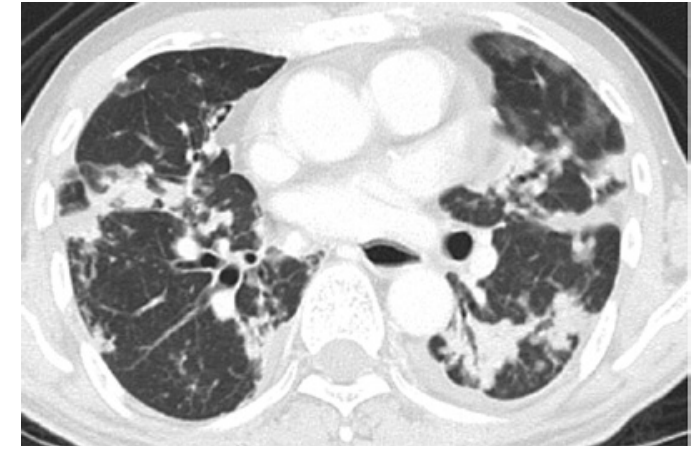

Figure 1. Chest computed tomography showing multiple nodules in the bilateral lungs.

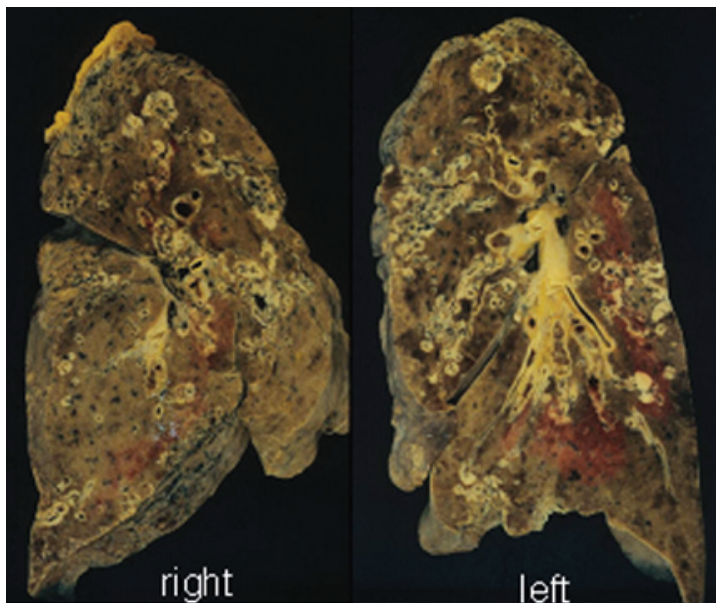

Figure 2. Cut surfaces of lung. Multiple nodules with central necrosis are present in the bilateral lungs.

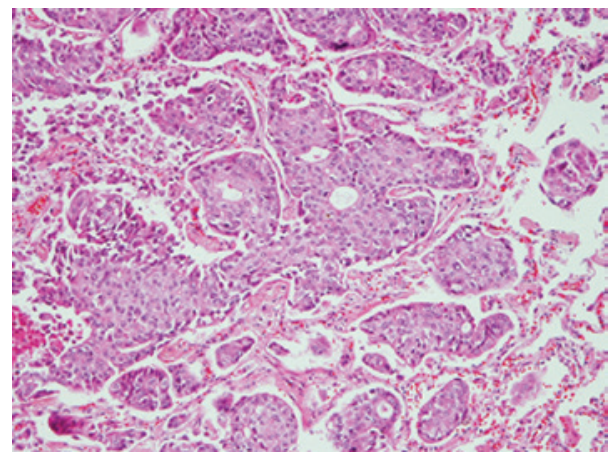

Figure 3. Histopathological findings of the lung nodule. Irregular tumor cell nests with ductal formation (H\&E stain; original magnification, x200).

\section{Results}

At autopsy, gross examination identified multiple yellowishwhite nodules with central necrosis, measuring $5 \mathrm{~mm}$ to $3 \mathrm{~cm}$, in the bilateral lungs (Fig. 2). Histopathological examination of the lung nodules showed infiltrative growth of variably sized and irregular-shaped epithelial cell nests with ductal formation (Fig. 3). These atypical epithelial cells had round to oval large nuclei with nucleolus and eosinophilic cytoplasm (Fig. 3). Alcian blue staining revealed intracytoplasmic mucin

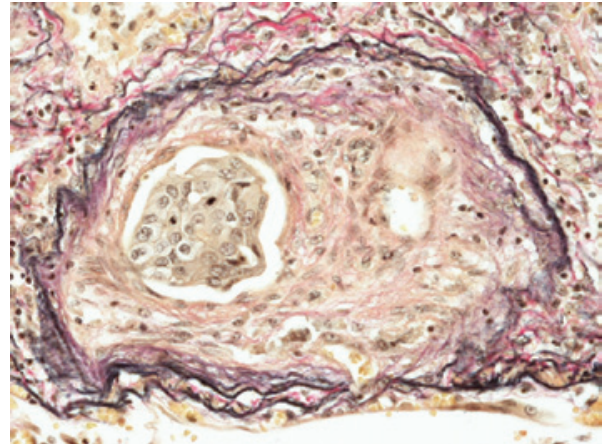

Figure 4. Fibrocellular intimal proliferation with tumor emboli (elastic van Gieson stain; original magnification, $\mathrm{x} 400$ ).

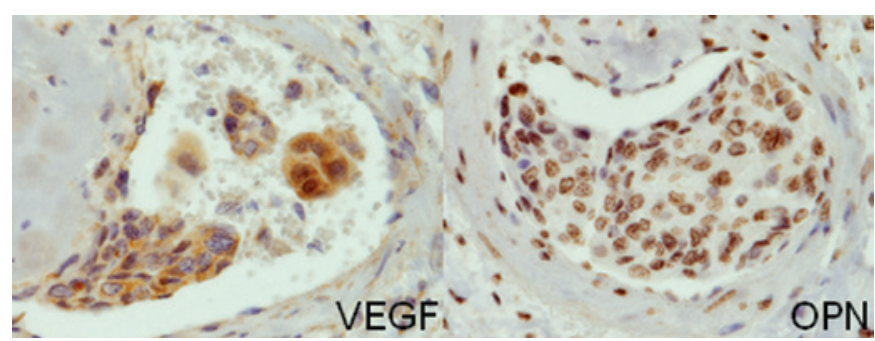

Figure 5. Immunohistochemical findings. Tumor cells in the vessel express VEGF and OPN (original magnification, $\mathrm{x} 400$ ).

Table I. Clinicopathological characteristics of pulmonary tumor thrombotic microangiopathy caused by lung cancer.

\begin{tabular}{lccc}
\hline Case no. & Age/Gender & Histology & Refs. \\
\hline 1 & $46 / \mathrm{M}$ & $\begin{array}{c}\text { Adenocarcinoma } \\
\text { (mucinous) }\end{array}$ & 1 \\
2 & $54 / \mathrm{M}$ & $\begin{array}{c}\text { Adenocarcinoma } \\
\text { (mucinous) }\end{array}$ & 1 \\
3 & $58 / \mathrm{M}$ & $\begin{array}{c}\text { Adenocarcinoma } \\
\text { (mucinous) }\end{array}$ & 1 \\
4 & $46 / \mathrm{F}$ & $\begin{array}{c}\text { Adenocarcinoma } \\
\text { Adenocarcinoma }\end{array}$ & 4 \\
$\begin{array}{l}\text { Present } \\
\text { case }\end{array}$ & $62 / \mathrm{M}$ & Adenocinom & \\
\hline
\end{tabular}

in the tumor cells. A poorly differentiated adenocarcinoma with multiple intrapulmonary metastases was diagnosed. The largest nodule was situated in the middle lobe of the right lung, which was thought to be the origin of the metastatic lesions.

Additionally, prominent fibrocellular and/or fibromuscular intimal proliferation of small arteries and arterioles with or without tumor emboli, which caused marked luminal stenosis, was identified (Fig. 4). These characteristic histopathological findings were consistent with PTTM caused by lung adenocarcinoma (1). Lymphatic tumor invasion was also observed.

Metastatic adenocarcinoma was identified in the liver, bilateral adrenal glands and kidneys, and lumbar vertebrae.

The immunohistochemical analyses revealed that the tumor cells were positive for VEGF and OPN (Fig. 5) 


\section{Discussion}

The suggested mechanism of PTTM involves tumor cells invading the pulmonary vascular system where they occlude the small arteries and arterioles, activate coagulation systems and release inflammatory mediators and growth factors. This process results in thrombosis, fibrocellular and/or fibromuscular intimal proliferation and luminal stenosis, which are the characteristic histopathological findings of PTTM and which differ from conventional vascular invasion by cancer cells $(1,2)$. Clinically, patients with PTTM often present with progressive dyspnea and severe pulmonary hypertension of unknown etiology, and develop acute cor pulmonale (1-3). Only four cases of PTTM caused by lung carcinoma have been reported $(1,4)$, and the histopathological typing of these cases is adenocarcinoma, as in the present case (Table I).

Studies have suggested that certain molecules produced by tumor cells are involved in the onset and/or development of PTTM $(2,3)$. One candidate is VEGF, a glycoprotein involved in the proliferation of endothelial cells and tumor angiogenesis. VEGF expression in tumor cells in cases of PTTM caused by gastric cancer have been reported $(2,3)$, and one case of PTTM caused by lung adenocarcinoma expressing VEGF has also been documented (4).

Another candidate is OPN, a phosphoglycoprotein produced by various carcinoma cells, which is known to promote adhesion, migration and proliferation of endothelial cells and fibroblasts (5). Takahashi et al speculated that OPN may promote intimal cell proliferation in cooperation with VEGF in the pathogenesis of PTTM caused by gastric cancer (3). This is the first reported case of PTTM caused by lung adenocarcinoma showing the possible involvement of both VEGF and OPN in promoting intimal proliferation and pathogenesis in PTTM.

PTTM rapidly progresses and is in most cases fatal since almost all patients with PTTM die within 1 week from the onset. Therefore, ante mortem diagnosis of PTTM is extremely difficult $(4,6)$. Miyano et al reported a notable case of PTTM caused by gastric cancer, which was diagnosed by lung biopsy. In their study, VEGF expression in tumor cells was confirmed by immunohistochemistry; the increased serum VEGF level became normalized after chemotherapy, resulting in a favorable clinical course (6). Their findings indicate that early clinical detection of PTTM may lead to timely and appropriate management as well as a more favorable prognosis.
PTTM is found in $3.3 \%$ of autopsies of patients with malignant tumors (1). The present case suggests that lung adenocarcinoma causes PTTM and shares a common mechanism with gastric adenocarcinoma. Therefore, it is necessary for both oncologists and pathologists to take particular note of PTTM.

\section{References}

1. Von Herbay A, Illes A, Waldherr R and Otto HF: Pulmonary tumor thrombotic microangiopathy with pulmonary hypertension. Cancer 66: 587-592, 1990.

2. Sakashita N, Yokose C, Fujii K, et al: Pulmonary tumor thrombotic microangiopathy resulting from metastatic signet ring cell carcinoma of the stomach. Pathol Int 57: 383-387, 2007.

3. Takahashi F, Kumasaka T, Nagaoka T, et al: Osteopontin expression in pulmonary tumor thrombotic microangiopathy caused by gastric carcinoma. Pathol Int 59: 752-756, 2009.

4. Uruga H, Morokawa N, Enomoto T, et al: A case of pulmonary tumor thrombotic microangiopathy associated with lung adenocarcinoma diagnosed by CT-guided lung biopsy. Nihon Kokyuki Gakkai Zasshi (In Japanese) 46: 928-933, 2008.

5. Denhardt DT, Noda M, O'Regan AW, Pavlin D and Berman JS: Osteopontin as a means to cope with environmental insults: regulation of inflammation, tissue remodeling, and cell survival. J Clin Invest 107: 1055-1061, 2001.

6. Miyano S, Izumi S, Takeda Y, et al: Pulmonary tumor thrombotic microangiopathy. J Clin Oncol 25: 597-599, 2007. 